\title{
Continuative statin therapy after percutaneous coronary intervention improves outcome in coronary bypass surgery: A propensity score analysis of 2501 patients
}

\author{
Vito Mannacio, MD, ${ }^{\mathrm{a}}$ Pascal Meier, MD, ${ }^{\mathrm{b}, \mathrm{c}}$ Anita Antignano, MD, ${ }^{\mathrm{d}}$ Michele Mottola, MD, ${ }^{\mathrm{a}}$ \\ Luigi Di Tommaso, MD, ${ }^{\mathrm{a}}$ Francesco Musumeci, MD, ${ }^{\mathrm{e}}$ and Carlo Vosa, $\mathrm{MD}^{\mathrm{a}}$
}

\begin{abstract}
Objectives: A history of percutaneous coronary intervention increases the risk of death and complications of coronary artery bypass grafting. This retrospective multicenter study evaluated the impact of continuative use of statin on postoperative outcomes when subsequent elective coronary artery bypass grafting is required after percutaneous coronary intervention.
\end{abstract}

\begin{abstract}
Methods: Among 14,575 patients who underwent isolated first-time coronary artery bypass grafting between January 2000 and December 2010, 2501 who had previous percutaneous coronary intervention with stenting and fulfilled inclusion criteria were enrolled. Continuative statin therapy was used in 1528 patients and not used in 973 patients. Logistic multiple regression and propensity score analyses were used to assess the risk-adjusted impact of statin therapy on in-hospital mortality and major adverse cardiac events. The Cox proportional hazards model was constructed to assess the effect of continuative statin therapy on 24-month outcome.
\end{abstract}

Results: At multivariate analysis, age more than 70 years, 3-vessel or 2-vessel plus left main coronary disease, multivessel percutaneous coronary intervention, ejection fraction 0.40 or less, diabetes mellitus, and logistic European System for Cardiac Operative Risk Evaluation 5 or greater were independent predictors of hospital mortality and major adverse cardiac events. After propensity score matching, conditional logistic regression analysis demonstrated that continuative statin therapy before coronary artery bypass grafting reduced the risk for hospital and 2-year mortality (odds ratio [OR], 0.27; 95\% confidence interval [CI], 0.12-0. 57; $P=.004$ and $\mathrm{OR}, 0.6 ; 95 \% \mathrm{CI}, 0.36-0.96 ; P=.04$, respectively) and major adverse cardiac events (OR, $0.31 ; 95 \%$ CI, $0.18-0.78 ; P=.003$ and $\mathrm{OR}, 0.5 ; 95 \% \mathrm{CI}, 0.34-0.76 ; P=.006$, respectively).

Conclusions: Long-term statin treatment after percutaneous coronary intervention improves early and midterm outcome when surgical revascularization will be required. (J Thorac Cardiovasc Surg 2014;148:1876-83)

See related commentary on pages 1884-6.

Recent studies indicate that $10 \%$ to $30 \%$ of the patients treated by percutaneous coronary intervention (PCI) for multivessel coronary disease require repeated coronary revascularization because of symptom recurrence and restenosis within 2 to 4 years after primary intervention. ${ }^{1,2}$ When a subsequent coronary artery bypass grafting $(\mathrm{CABG})$ is necessary, a history of PCI is associated with a higher incidence of perioperative adverse events. ${ }^{3-7}$ Thus,

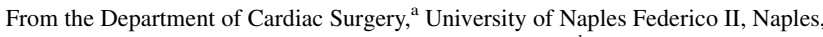
Italy; Divisions of Cardiology and Cardiac Surgery, ${ }^{\mathrm{b}}$ Yale Medical School, New Haven, Conn; Cardiology, ${ }^{\mathrm{c}}$ University College London Hospital, London, United Kingdom; Department of Cardiology, ${ }^{d}$ Azienda Ospedaliera SantobonoPausillipon, Naples, Italy; and Department of Cardiac Surgery, ${ }^{\mathrm{e}}$ Azienda Ospedaliera S. Camillo Forlanini, Rome, Italy.

Disclosures: Authors have nothing to disclose with regard to commercial support.

Received for publication Dec 18, 2013; revisions received Feb 4, 2014; accepted for publication Feb 14, 2014; available ahead of print March 22, 2014.

Address for reprints: Vito Mannacio, MD, Via S. Domenico 62, 80127 Naples, Italy (E-mail: vitomannacio2@libero.it).

$0022-5223 / \$ 36.00$

Copyright (c) 2014 by The American Association for Thoracic Surgery

http://dx.doi.org/10.1016/j.jtcvs.2014.02.045
}

in the setting of these high-risk patients, novel technical and pharmacologic strategies are required.

Recent guidelines suggest antiplatelet therapy for primary and secondary prevention before and after implantation of stents or in surgical patients to improve vein-graft patency after CABG. ${ }^{8-11}$ Furthermore, robust evidence supports the early and aggressive therapy with inhibitors of 3-hydroxy-3-methylglutaryl coenzyme A reductase statins to reduce cardiovascular adverse events and repeat revascularization procedures after PCI. ${ }^{12}$ In patients undergoing $\mathrm{CABG}$, statin therapy exerts multiple pleiotropic effects associated with reduction of myocardial damage and better surgical results. ${ }^{13-17}$ This overall efficacy of statins is reflected in the latest American College of Cardiology/American Heart Association guidelines. However, the effect of statin on postoperative outcome of patients with a history of PCI, subsequently referred to $\mathrm{CABG}$, has not been evaluated until now.

Because of the important clinical implications, this study evaluated the impact of continuative statin treatment on early and midterm cardiac mortality and nonfatal major adverse cardiac events (MACE) in those patients who are finally referred to elective CABG after previous PCI by stenting. 

Abbreviations and Acronyms
$\mathrm{CABG}=$ coronary artery bypass grafting
$\mathrm{CI}=$ confidence interval
$\mathrm{CPB}=$ cardiopulmonary bypass
MACE $=$ major adverse cardiac events
MI = myocardial infarction
NO $=$ nitric oxide
$\mathrm{OR} \quad=$ odds ratio
PCI = percutaneous coronary intervention

\section{PATIENTS AND METHODS}

The study was a retrospective multicenter cohort study designed according to the STROBE statement. Among a total of 14,575 patients who underwent first-time isolated CABG between January 2000 and February 2010, 2051 consecutive patients previously treated by PCI by stenting were evaluated. Additional cardiac procedures and urgent or emergency CABG were exclusion criteria. Patients were classified depending on whether they received (statin group) or did not receive (no statin group) continuous treatment with any kind of statins during the period between PCI and CABG surgery. Types of statin used and the usual dosages were as follows: atorvastatin 20 to $40 \mathrm{mg} / \mathrm{d}$, rosuvastatin 10 to $20 \mathrm{mg} / \mathrm{d}$, simvastatin 20 to $40 \mathrm{mg} / \mathrm{d}$, pravastatin 20 to $40 \mathrm{mg} / \mathrm{d}$, and fluvastatin 20 to $40 \mathrm{mg} / \mathrm{d}$. All patients with suspected low adherence to statin therapy during the last 6 months before CABG were excluded. Adherence to therapy was assessed by rates of prescription refills as reported by Osterberg and Blaschke. ${ }^{18}$ We measured statin adherence as the proportion of days covered. We defined patients as being "adherent" if the proportion of days covered was $80 \%$ or more. Change from one statin to another was considered adherence with therapy. A total of 1528 patients treated by continuative statin therapy were identified and compared with 973 patients who did not receive continuative statin treatment after PCI. In the statin group, the overall proportion of days covered was $86.4 \%$.

All patients underwent preoperative coronary angiography. Risk stratification was assessed by the logistic European System for Cardiac Operative Risk Evaluation. Low-density lipoprotein levels before the operation were $120 \pm 28 \mathrm{mg} / \mathrm{dL}$ in the statin group and $159 \pm 39 \mathrm{mg} / \mathrm{dL}$ in the no statin group $(P<.001)$. The main demographic and clinical characteristics of the patients are shown in Table 1.

Troponin I values, assessed preoperatively, and at 8 and 12 hours after operation, and then on every postoperative day until hospital discharge, were collected as markers of myocardial damage. The dose of inotropic support, when required, was considered indicative of postoperative outcome. To avoid bias due to the different duration of follow-up, the last time point for postoperative evaluation was fixed at 24 postoperative months in all patients.

The study protocol was approved by the ethics committee of the University of Naples Federico II. All patients preliminarily granted permission for the use of their medical records for research purposes; thus, individual patient consent was waived for this study.

\section{Perioperative Management}

Patients receiving antiplatelet therapy before surgery were managed in accordance with the 2011 American College of Cardiology Foundation/American Heart Association Guideline for Coronary Artery Bypass Graft Surgery. ${ }^{19}$ Standardized dual antiplatelet therapy was started when chest tube drainage was less than $20 \mathrm{~mL} / \mathrm{h} .{ }^{9}$ Statins were given 24 hours after surgery and continued over all follow-up time in all patients. ${ }^{16}$

\section{Outcome Measures and Definitions}

The end points were (1) hospital mortality and major adverse cardiac events (MACE) and (2) 2-year mortality and MACE.

Hospital mortality was defined as death within 30 days or at any time after operation if the patient did not leave the hospital alive. MACE were defined as a composite end point of nonfatal perioperative myocardial infarction (MI), low output syndrome, significant cardiac arrhythmias, and need for repeat surgical or percutaneous revascularization.

Perioperative acute MI was diagnosed when the criteria indicated by the Joint European Society of Cardiology/American College of Cardiology Foundation/American Heart Association/World Heart Federation Task Force for the Universal Definition of Myocardial Infarction guidelines were fulfilled. ${ }^{20}$ Troponin I leakage without electrocardiographic modifications, new loss of viable myocardium, and new regional wall motion abnormality were considered as myocardial damage. ${ }^{16}$ Postoperative renal disease was defined as serum creatinine $2.5 \mathrm{mg} / \mathrm{dL}$ or greater. Low output syndrome was diagnosed as previously described. ${ }^{21}$ High-dose inotropic support was defined when $7 \mu \mathrm{g} \cdot \mathrm{kg}^{-1} \cdot \mathrm{min}^{-1}$ or greater of dopamine or any dose of adrenaline was added.

\section{Statistical Analysis}

Descriptive statistics were summarized for categoric variables as percentages and compared using the chi-square exact test. Univariate logistic regressions were performed to identify preoperative independent predictors for hospital mortality and MACE based on the preoperative variables mentioned in Table 1. Multivariate logistic regression model included those variables with a probability value of .05 or less for association with at least 1 study end point.

To eliminate confounding bias due to unequal distribution of risk factors among groups, propensity score was performed to generate a subset of matched cases (statin therapy) and controls (no statin therapy) who had the same distribution of covariates. SAS/STAT logistic procedure and a SAS \%GMATCH Macro program (SAS Institute Inc, Cary, NC) were used to match cases to controls. Variables listed in Table 1 were used to build a fully adjusted multivariable logistic regression model to compute a propensity score for each patient. The cases were matched with controls to create a 1-to-1 match. Matching was done without replacement, based on caliper matching within a prespecified distance of a maximum of 0.2 of the standard deviation of the logit of the propensity score. If more than 1 control matched equally to 1 case, the control was selected at random.

Comparisons between the 2 groups were performed by the chi-square test to confirm that the 2 groups were successfully matched. The $\mathrm{c}$ statistic was 0.89 , indicating the good discriminatory power of the propensity model. Hospital mortality and MACE were compared between the 2 matched groups by the 2-tailed McNemar test. Multi-way analysis of variance with correction for serial measurements analyzed troponin I, inotropic support, and postoperative creatinine levels. A Cox proportional hazards model was constructed using the variables reported in Table 1 to evaluate the effect of continuative statin therapy on 24-month survival and MACE. All statistical analyses were performed with the SAS system, version 9.1 (SAS Institute Inc) or SPSS version 13.0 for Windows (SPSS Inc, Chicago, Ill).

\section{RESULTS}

All 2501 patients underwent CABG at a median of 38 months (interquartile range, 29-48) after a mean of 1.9 angioplasties by stenting. A total of 1211 patients $(48.4 \%)$ underwent operation with cardiopulmonary bypass (CPB), and 1290 patients $(51.6 \%)$ underwent operation off-pump. In patients who underwent on-pump CABG, mean $\mathrm{CPB}$ and mean aortic crossclamp times were $83.5 \pm 25.2$ minutes and $43.2 \pm 13.2$ minutes, 
TABLE 1. Main clinical and demographic characteristics

\begin{tabular}{|c|c|c|c|c|c|}
\hline & \multicolumn{2}{|c|}{ Statin group (1528) } & \multicolumn{2}{|c|}{ No statin group (973) } & \multirow[b]{2}{*}{$P$ value } \\
\hline & No. of patients & $\%$ & No. of patients & $\%$ & \\
\hline Age $\geq 70 y$ & 534 & 35 & 311 & 32 & .06 \\
\hline Female sex & 382 & 25 & 272 & 28 & .1 \\
\hline BMI & 275 & 18 & 205 & 21 & .06 \\
\hline Hypertension & 886 & 58 & 535 & 55 & .1 \\
\hline Hypercholesterolemia & 413 & 27 & 370 & 35 & $<.001$ \\
\hline LDL cholesterol $\geq 120 \mathrm{mg} / \mathrm{dL}$ & 395 & 26 & 358 & 37 & $<.001$ \\
\hline Diabetes mellitus & 581 & 38 & 331 & 34 & .04 \\
\hline Peripheral vascular disease & 244 & 16 & 116 & 12 & .005 \\
\hline Respiratory disease & 367 & 24 & 224 & 23 & .6 \\
\hline Renal disease & 137 & 9 & 78 & 8 & .4 \\
\hline History of MI & 718 & 47 & 399 & 41 & .003 \\
\hline Ejection fraction $\leq 40 \%$ & 245 & 16 & 146 & 15 & .5 \\
\hline NYHA class $\geq$ III & 580 & 38 & 341 & 35 & .1 \\
\hline Logistic euroSCORE $\geq 5$ & 458 & 30 & 253 & 26 & .03 \\
\hline Multivessel coronary disease* & 1008 & 66 & 613 & 63 & .1 \\
\hline Left main disease & 304 & 20 & 214 & 22 & .2 \\
\hline Single previous PCI & 993 & 64 & 678 & 69 & .01 \\
\hline Multiple previous PCI & 535 & 35 & 295 & 30 & .01 \\
\hline \multicolumn{6}{|l|}{ Type of stent } \\
\hline Bare-metal & 688 & 45 & 506 & 52 & $<.001$ \\
\hline Drug-eluting & 840 & 55 & 467 & 48 & $<.001$ \\
\hline \multicolumn{6}{|l|}{ Indications for $\mathrm{CABG}$} \\
\hline In-stent restenosis & 504 & 33 & 294 & 30 & .1 \\
\hline De novo stenosis & 456 & 30 & 327 & 33 & 6 \\
\hline Combined & 539 & 35 & 332 & 35 & .8 \\
\hline In-stent thrombosis & 29 & 2 & 20 & 2 & .9 \\
\hline$\geq 3$ grafts/patient & 965 & 63 & 576 & 59 & .1 \\
\hline Off-pump CABG & 792 & 52 & 498 & 51 & .8 \\
\hline On-pump CABG & 736 & 48 & 475 & 49 & .8 \\
\hline Aortic crossclamp time $>80 \mathrm{~min}$ & 271 & 18 & 145 & 15 & .7 \\
\hline \multicolumn{6}{|l|}{ Therapy } \\
\hline Dual antiplatelet & 1329 & 87 & 827 & 85 & .1 \\
\hline Single antiplatelet (ASA) & 199 & 13 & 146 & 15 & .1 \\
\hline Insulin & 81 & 5 & 50 & 5 & .9 \\
\hline Oral hypoglycemic & 506 & 33 & 287 & 29 & .06 \\
\hline$\beta$-blockers & 1100 & 72 & 671 & 69 & .1 \\
\hline Calcium antagonists & 488 & 32 & 301 & 31 & 6 \\
\hline ACE inhibitors & 352 & 23 & 204 & 21 & .2 \\
\hline
\end{tabular}

ACE, Angiotensin-converting enzyme; ASA, acetylsalicylic acid; $B M I$, body mass index; $C A B G$, coronary artery bypass grafting; euroSCORE, European System for Cardiac Operative Risk Evaluation; $L D L$, low-density lipoprotein; $M I$, myocardial infarction; $N Y H A$, New York Heart Association; $P C I$, percutaneous coronary intervention.

*Multivessel coronary disease was intended as 3-vessel or 2-vessel plus left main disease.

respectively, without significant differences between groups $(86.4 \pm 25.3$ vs $84.7 \pm 30.1, P=.07$ and $43.8 \pm 12.3$ vs $41.6 \pm 15.2, P=.06)$. As a general rule, on-pump CABG did not result in additional risks for hospital death or MACE compared with off-pump CABG. However, on multivariate analysis of the entire population of patients who underwent on-pump operation, the risk of hospital deaths and MACE was significantly increased in those patients who had a prolonged aortic crossclamp time ( $>80$ minutes) $(P=.01$ and $P=.01$, respectively). In this subpopulation, statin-treated patients had an odds of death $25 \%(95 \%$ CI, $0.25-0.95 ; P=.03)$ and MACE $30 \%$
(95\% CI, 0.12-0.8; $P=.03$ ) lower than in statin-naive patients.

The number of conduits implanted was 4278 in the statin group and 2530 in the no statin group. The average number of grafts for patient was higher in the statin group $(2.8 \pm 0.5$ vs $2.6 \pm 0.6, P<.001$ ). The index of completeness (the number of grafts effectively performed on the number of grafts intended at angiography) was similar (0.95 vs 0.94 , $P=.8$ ), as well as the use of the left internal thoracic artery $(1473 / 1528 ; 96 \%$ vs $946 / 973 ; 97 \%, P=.1)$. Other arterial conduits (right internal thoracic artery or radial artery) were used in $65 \%$ of patients in the statin group (993/1528) and 
TABLE 2. Univariate and multivariate logistic regressions for hospital death

\begin{tabular}{|c|c|c|c|c|}
\hline \multirow[b]{2}{*}{ Characteristic } & \multicolumn{2}{|c|}{ Univariate } & \multicolumn{2}{|c|}{ Multivariate } \\
\hline & OR $(95 \%$ CI $)$ & $P$ value & OR $(95 \%$ CI $)$ & $P$ value \\
\hline Age $\geq 70$ y & $2.5(1.0-5.3)$ & .02 & $2.4(1.0-4.1)$ & .04 \\
\hline Female sex & $1.5(0.6-3.5)$ & .3 & & \\
\hline $\mathrm{BMI} \geq 30$ & $2.4(0.9-6.5)$ & .07 & & \\
\hline Hypertension & $3.1(1.5-6.2)$ & $<.001$ & $2.2(1.3-4.6)$ & .008 \\
\hline Hypercholesterolemia & $2.8(1.2-6.7)$ & .01 & $1.5(0.6-3.4)$ & .09 \\
\hline LDL cholesterol $\geq 120 \mathrm{mg} / \mathrm{dL}$ & $1.7(0.9-3.1)$ & .05 & & \\
\hline Diabetes mellitus & $3.1(1.4-7.1)$ & .003 & $2.8(1.0-5.2)$ & .02 \\
\hline Peripheral vascular disease & $5.4(1.8-15.3)$ & .005 & $3.4(1.4-9.7)$ & .02 \\
\hline Respiratory disease & $3.4(1.0-10.8)$ & .003 & $2.1(0.7-7.2)$ & .08 \\
\hline Renal disease & $4.3(1.1-15.5)$ & .002 & $1.6(0.8-11.1)$ & .1 \\
\hline History of MI & $4.3(1.9-9.9)$ & $<.001$ & $2.9(1.5-6.1)$ & .002 \\
\hline Ejection fraction $\leq 40 \%$ & $4.2(1.4-12.6)$ & .004 & $2.1(1.0-6.7)$ & .02 \\
\hline NYHA class $\geq$ III & $3.3(1.3-8.1)$ & .004 & $2.2(0.8-5.5)$ & .09 \\
\hline Logistic euroSCORE $\geq 5$ & $2.8(1.3-5.8)$ & .003 & $1.3(1.1-4.1)$ & .01 \\
\hline Multivessel coronary disease* & $2.3(1.2-4.5)$ & .008 & $2.0(1.1-3.1)$ & .01 \\
\hline Left main disease $\geq 50 \%$ & $1.9(0.9-4.5)$ & .1 & & \\
\hline Single previous PCI & $1.9(0.8-4.4)$ & .1 & & \\
\hline Multiple previous PCI & $3.0(1.3-6.3)$ & .004 & $2.1(1.1-4.4)$ & .02 \\
\hline \multicolumn{5}{|l|}{ Type of stent } \\
\hline Bare-metal stent & $2.1(0.9-4.9)$ & .07 & & \\
\hline Drug-eluting stent & $2.5(1.1-5.6)$ & .01 & $1.8(0.6-4.1)$ & .1 \\
\hline \multicolumn{5}{|l|}{ Indications for $\mathrm{CABG}$} \\
\hline In-stent restenosis & $1.1(0.2-5.5)$ & .8 & & \\
\hline De novo stenosis & $2.7(0.9-8.5)$ & .7 & & \\
\hline Combined & $2.2(0.8-6.5)$ & .1 & & \\
\hline In-stent thrombosis & $2.6(0.7-9.5)$ & .1 & & \\
\hline$\geq 3$ grafts/patients & $1.8(0.9-3.4)$ & .05 & & \\
\hline Off-pump CABG & $1.7(0.7-4.2)$ & .2 & & \\
\hline On-pump CABG & $2.3(1.0-4.9)$ & .02 & $2.0(0.8-3.2)$ & .06 \\
\hline Aortic crossclamp time $>80 \mathrm{~min}$ & $2.4(1.3-3.9)$ & .008 & $2.1(1.1-3.2)$ & .01 \\
\hline \multicolumn{5}{|l|}{ Postoperative variables } \\
\hline High-dose inotropic support & $2.4(1.0-5.7)$ & .03 & $1.8(0.7-3.8)$ & .09 \\
\hline Significant troponin I leakage & $1.1(0.4-2.9)$ & .9 & & \\
\hline \multicolumn{5}{|l|}{ Therapy } \\
\hline Dual antiplatelet & $2.0(0.9-4.2)$ & .05 & & \\
\hline Single antiplatelet (ASA) & $2.5(1.0-6.5)$ & .03 & $1.9(0.6-4.1)$ & .08 \\
\hline Insulin & $2.5(1.1-5.9)$ & .04 & $2.1(0.7-4.2)$ & .08 \\
\hline Oral hypoglycemic & $1.2(0.5-1.4)$ & .8 & & \\
\hline$\beta$-blockers & $1.1(0.6-1.4)$ & .7 & & \\
\hline Calcium antagonists & $1.1(0.5-1.2)$ & .8 & & \\
\hline ACE inhibitors & $1.4(0.5-2.0)$ & .9 & & \\
\hline
\end{tabular}

$\overline{A C E}$, Angiotensin-converting enzyme; $A S A$, acetylsalicylic acid; $B M I$, body mass index; $C A B G$, coronary artery bypass grafting; $C I$, confidence interval; euroSCORE, European System for Cardiac Operative Risk Evaluation; $L D L$, low-density lipoprotein; $M I$, myocardial infarction; NYHA, New York Heart Association; $O R$, odds ratio; PCI, percutaneous coronary intervention. *Multivessel coronary disease was intended as 3-vessel or 2-vessel plus left main disease.

in $59 \%$ of patients in the no statin group (574/973) $(P=.003)$.

Total unadjusted hospital mortality was $2.3 \%(59 / 2501$ patients). Mortality was lower in the statin group $(24 / 1528 ; 1.5 \%$ vs $35 / 973 ; 3.3 \% ; P=.001)$. Postoperative MACE occurred more frequently in the no statin group (79/1528; $5.1 \%$ vs 76/973; $7.8 \%, P=.009)$. Univariate and multivariate logistic regression analyses for hospital deaths and MACE revealed several variables as independent predictors for hospital deaths (Table 2) and MACE (Table 3).

In agreement with the concept that preoperative statin therapy protects renal function after coronary surgery, mean serum creatinine levels were significantly increased compared with preoperative values in the no statin group (from $0.82 \pm 0.22$ to $0.85 \pm 0.25, P=.006$ ) than in the statin group (from $0.82 \pm 0.19$ to $0.83 \pm 0.23$, $P=.2$ ). 
TABLE 3. Univariate and multivariate logistic regressions for major adverse cardiac events

\begin{tabular}{|c|c|c|c|c|}
\hline \multirow[b]{2}{*}{ Characteristic } & \multicolumn{2}{|c|}{ Univariate } & \multicolumn{2}{|c|}{ Multivariate } \\
\hline & OR $(95 \%$ CI $)$ & $P$ value & OR $(95 \%$ CI $)$ & $P$ value \\
\hline Age $\geq 70 y$ & $2.3(1.4-3.7)$ & $<.001$ & $1.8(1.2-3.3)$ & .01 \\
\hline Female sex & $1.3(0.8-2.2)$ & .2 & & \\
\hline $\mathrm{BMI} \geq 30$ & $1.0(0.9-1.9)$ & .9 & & \\
\hline Hypertension & $1.8(1.2-2.8)$ & .003 & $1.4(1.0-2.1)$ & .03 \\
\hline Hypercholesterolemia & $1.6(1.0-2.7)$ & .04 & $1.2(0.7-2.4)$ & .07 \\
\hline LDL cholesterol $\geq 120 \mathrm{mg} / \mathrm{dL}$ & $1.3(0.7-2.4)$ & .2 & $1.1(1.0-2.2)$ & .05 \\
\hline Diabetes mellitus & $2.9(1.8-4.8)$ & $<.001$ & $2.2(1.3-4.1)$ & .004 \\
\hline Peripheral vascular disease & $3.5(2.0-6.1)$ & $<.001$ & $2.8(1.5-4.7)$ & .002 \\
\hline Respiratory disease & $1.4(0.7-2.7)$ & .4 & & \\
\hline Renal disease & $1.6(0.8-3.3)$ & .1 & & \\
\hline History of MI & $2.7(1.6-4.5)$ & $<.001$ & $1.9(1.1-3.1)$ & .008 \\
\hline Ejection fraction $\leq 40 \%$ & $2.0(1.1-3.6)$ & .01 & $1.8(1.0-3.1)$ & .04 \\
\hline NYHA class $\geq$ III & $1.7(1.0-2.8)$ & .02 & $1.4(0.8-2.1)$ & .1 \\
\hline Logistic euroSCORE $\geq 5$ & $2.1(1.3-3.4)$ & $<.001$ & $1 . .9(1.1-3.0)$ & .007 \\
\hline Multivessel coronary disease* & $1.8(1.2-2.6)$ & .001 & $1.4(1.0-2.3)$ & .03 \\
\hline Left main disease $\geq 50 \%$ & $1.6(0.9-2.6)$ & .05 & & \\
\hline Single previous PCI & $1.4(0.7-2.7)$ & .3 & & \\
\hline Multiple previous PCI & $1.9(1.2-2.9)$ & .001 & $1.6(0.7-2.4)$ & .08 \\
\hline \multicolumn{5}{|l|}{ Type of stent } \\
\hline Bare-metal stent & $1.4(0.9-2.2)$ & .1 & & \\
\hline Drug-eluting stent & $1.6(1.0-2.7)$ & .04 & $1.4(0.6-2.1)$ & .1 \\
\hline \multicolumn{5}{|l|}{ Indications for $\mathrm{CABG}$} \\
\hline In-stent restenosis & $1.9(1.0-3.7)$ & .03 & $1.2(0.5-2.7)$ & .1 \\
\hline De novo stenosis & $1.7(0.9-3.4)$ & .1 & & \\
\hline Combined & $1.3(0.7-2.5)$ & .5 & & \\
\hline In-stent thrombosis & $1.4(0.6-3.4)$ & .4 & & \\
\hline$\geq 3$ grafts/patient & $1.4(1.0-2.1)$ & .04 & $1.3(0.7-1.8)$ & .1 \\
\hline Off-pump CABG & $1.4(0.8-2.5)$ & .2 & & \\
\hline On-pump CABG & $1.6(1.0-2.4)$ & .03 & $1.4(07-2.1)$ & .09 \\
\hline Aortic crossclamp time $>80 \mathrm{~min}$ & $1.7(1.1-2.6)$ & .004 & $1.4(1.0-2.1)$ & .01 \\
\hline \multicolumn{5}{|l|}{ Postoperative variables } \\
\hline High-dose inotropic support & $1.3(0.7-2.5)$ & .3 & & \\
\hline Significant troponin I leakage & $2.1(1.2-3.8)$ & .004 & $1.8(1.1-3.3)$ & .009 \\
\hline \multicolumn{5}{|l|}{ Therapy } \\
\hline Dual antiplatelet & $1.2(0.7-2.2)$ & .3 & & \\
\hline Single antiplatelet (ASA) & $1.7(1.0-2.8)$ & .03 & $1.4(0.7-2.1)$ & .09 \\
\hline Insulin & $2.2(1.3-3.8)$ & .004 & $1.9(1.2-3.1)$ & .01 \\
\hline Oral hypoglycemic & $1.2(0.6-2.8)$ & .2 & & \\
\hline$\beta$-blockers & $1.1(0.5-2.5)$ & .2 & & \\
\hline Calcium antagonists & $1.4(0.8-2.6)$ & .3 & & \\
\hline ACE inhibitors & $1.6(0.7-2.9)$ & .2 & & \\
\hline
\end{tabular}

$\overline{A C E}$, Angiotensin-converting enzyme; $A S A$, acetylsalicylic acid; $B M I$, body mass index; $C A B G$, coronary artery bypass grafting; $C I$, confidence interval; euroSCORE, European System for Cardiac Operative Risk Evaluation; $L D L$, low-density lipoprotein; $M I$, myocardial infarction; NYHA, New York Heart Association; $O R$, odds ratio; $P C I$, percutaneous coronary intervention. *Multivessel coronary disease was intended as 3-vessel or 2-vessel plus left main disease.

Of note, the subgroup analysis of patients who had been treated by PCI for the left anterior descending artery and patients who had been treated for a coronary artery other than the left anterior descending artery showed no differences in hospital mortality $(P=.7)$ and MACE $(P=.4)$. Likewise, patients with a history of single PCI had no significant differences between groups in terms of hospital deaths $(12 / 993 ; 1.2 \%$ in the statin group vs $10 / 678 ; 1.4 \%$ in the no statin group, $P=.8$ ) or MACE
(33/993; $3.3 \%$ in the statin group vs $31 / 678 ; 4.5 \%$ in the no statin group, $P=.2$ ). By contrast, a significant reduction of hospital deaths and MACE occurred in the statin group when subgroups of patients with a history of multiple PCI were compared $(12 / 535 ; 2.2 \%$ vs $25 / 295 ; 8.4 \%, P<.001$ and $46 / 535 ; 8.4 \%$ vs $45 / 295 ; 15.2 \%, P=.004)$.

A high dose of inotropic support postoperatively was required by 152 patients $(9.9 \%)$ in the statin group versus 144 patients $(14.7 \%)$ in the no statin group $(P<.001)$. 
TABLE 4. Main clinical and demographic patient characteristics in subgroups matched on propensity score

\begin{tabular}{|c|c|c|c|c|c|}
\hline & \multicolumn{2}{|c|}{ Statin group (931) } & \multicolumn{2}{|c|}{ No statin group (931) } & \multirow[b]{2}{*}{$P$ value } \\
\hline & No. of patients & $\%$ & No. of patients & $\%$ & \\
\hline Age $\geq 70 y$ & 303 & 32 & 292 & 31 & .6 \\
\hline Female sex & 251 & 27 & 257 & 27 & .8 \\
\hline BMI & 199 & 21 & 191 & 20 & .6 \\
\hline Hypertension & 538 & 58 & 521 & 56 & .4 \\
\hline Hypercholesterolemia & 371 & 40 & 361 & 39 & 6 \\
\hline LDL cholesterol $\geq 120 \mathrm{mg} / \mathrm{dL}$ & 348 & 37 & 351 & 38 & .07 \\
\hline Diabetes mellitus & 335 & 36 & 331 & 35 & .9 \\
\hline Peripheral vascular disease & 123 & 13 & 113 & 12 & .8 \\
\hline Respiratory disease & 220 & 24 & 222 & 24 & .9 \\
\hline Renal disease & 85 & 9 & 78 & 8 & .6 \\
\hline History of MI & 415 & 44 & 398 & 42 & .4 \\
\hline Ejection fraction $\leq 40 \%$ & 141 & 15 & 135 & 14 & .8 \\
\hline NYHA class $\geq$ III & 341 & 36 & 331 & 35 & .6 \\
\hline Logistic euroSCORE $\geq 5$ & 245 & 26 & 239 & 25 & .8 \\
\hline Multivessel coronary disease ${ }^{*}$ & 615 & 66 & 601 & 64 & .5 \\
\hline Left main disease & 201 & 22 & 203 & 22 & .9 \\
\hline Single previous PCI & 644 & 69 & 650 & 70 & .8 \\
\hline Multiple previous PCI & 287 & 31 & 281 & 30 & .8 \\
\hline \multicolumn{6}{|l|}{ Type of stent } \\
\hline Bare-metal & 469 & 50 & 483 & 52 & .5 \\
\hline Drug-eluting & 462 & 50 & 448 & 48 & .5 \\
\hline \multicolumn{6}{|l|}{ Indications for $\mathrm{CABG}$} \\
\hline In-stent restenosis & 279 & 30 & 285 & 30 & .9 \\
\hline De novo stenosis & 310 & 33 & 317 & 34 & .7 \\
\hline Combined & 327 & 35 & 312 & 34 & .7 \\
\hline In-stent thrombosis & 15 & 2 & 17 & 2 & .9 \\
\hline$\geq 3$ grafts/patient & 585 & 63 & 550 & 59 & .6 \\
\hline Off-pump CABG & 483 & 52 & 473 & 51 & .6 \\
\hline On-pump CABG & 448 & 48 & 458 & 49 & .6 \\
\hline Aortic crossclamp time $>80 \mathrm{~min}$ & 142 & 15 & 137 & 14 & .8 \\
\hline \multicolumn{6}{|l|}{ Therapy } \\
\hline \multicolumn{6}{|l|}{ Dual antiplatelet } \\
\hline Single antiplatelet (ASA) & 121 & 13 & 137 & 15 & .3 \\
\hline Insulin & 33 & 3 & 36 & 3 & .9 \\
\hline Oral hypoglycemic & 288 & 31 & 270 & 29 & .4 \\
\hline$\beta$-blockers & 670 & 72 & 670 & 69 & .9 \\
\hline Calcium antagonists & 281 & 30 & 288 & 31 & .7 \\
\hline ACE inhibitors & 216 & 23 & 203 & 22 & .5 \\
\hline
\end{tabular}

ACE, Angiotensin-converting enzyme; ASA, acetylsalicylic acid; BMI, body mass index; CABG, coronary artery bypass grafting; euroSCORE, European System for Cardiac Operative Risk Evaluation; $L D L$, low-density lipoprotein; $M I$, myocardial infarction; NYHA, New York Heart Association; PCI, percutaneous coronary intervention.

*Multivessel coronary disease was intended as 3-vessel or 2-vessel plus left main disease.

Significant postoperative increases in troponin I values occurred in the no statin group $(61.9 \%$ vs $37.8 \%$, $P<.001)$. Mean postoperative peak value of troponin I was lower in the statin group $(0.21 \pm 0.18 \mathrm{ng} / \mathrm{mL}$ vs 0.42 $\pm 0.38 \mathrm{ng} / \mathrm{mL} ; P<.001$ ). Troponin I levels peaked at 12 hours after the operation in the statin group and at 8 hours in the no statin group.

After propensity score matching, 931 cases were successfully 1:1 matched with 931 corresponding controls (Table 4). After matching, the average number of grafts for patients was similar in both groups $(2.72 \pm 0.8 \mathrm{vs}$ $2.66 \pm 0.5, P=.05)$, as well as the number of left thoracic arteries implanted (914 in the statin group vs 917 in the no statin group, $P=1$ ). Other arterial conduits (right internal thoracic artery or radial artery) were used in $63 \%$ of the statin group $(591 / 931)$ and in $59 \%$ of the no statin group $(550 / 931)(P=.06)$. Postoperative hospitalization was significantly lower in the statin group $(10.2 \pm 2.9$ days vs $9.2 \pm 2.9$ days, $P<.001)$. Hospital mortality was $1.9 \%$ (18 of 931) in the statin group versus $3.7 \%(35 / 931)$ in the no statin group $(P=.02)$, and the MACE rate was 5.5\% (52/931) versus $9.1 \%(85 / 931)$, respectively $(P=.03)$. The McNemar test for paired proportion confirmed significant differences in hospital 
TABLE 5. Two-year mortality and major adverse cardiac events in propensity score matched patients analyzed by Cox regression model

\begin{tabular}{lccc}
\hline \multicolumn{1}{c}{ Variables } & $\boldsymbol{P}$ value & Exp & $\mathbf{9 5 \%}$ CI \\
\hline Mortality & & & \\
Ejection fraction $\leq 0.40$ & .004 & 1.7 & $1.3-2.9$ \\
Multiple previous PCI & $<.001$ & 2.4 & $1.5-3.6$ \\
Multivessel coronary disease* & .006 & 2.1 & $1.7-2.6$ \\
Logistic euroSCORE $\geq 5$ & .004 & 2.3 & $1.8-3.4$ \\
Perioperative troponin I leakage & $<.001$ & 3.1 & $2.1-3.8$ \\
No statin therapy before CABG & .002 & 3.0 & $2.0-3.6$ \\
MACE & & & \\
Female sex & .02 & 1.8 & $1.2-3.1$ \\
Diabetes mellitus & .02 & 1.9 & $1.3-3.5$ \\
Peripheral vascular disease & .009 & 2.1 & $1.4-4.1$ \\
Multiple previous PCI & $<.001$ & 2.7 & $1.9-3.9$ \\
Logistic euroSCORE $\geq 5$ & $<.001$ & 2.7 & $1.8-3.9$ \\
Multivessel coronary disease* & .002 & 2.4 & $2.1-3.5$ \\
Perioperative troponin I leakage & $<.001$ & 3.1 & $2.1-4.5$ \\
No statin therapy before CABG & $<.001$ & 3.4 & $2.2-4.6$ \\
Single antiplatelet (ASA) & .008 & 2.1 & $1.4-3.9$ \\
\hline
\end{tabular}

Variables tested: demographic and clinical variables reported in Table 1. $A S A$, Acetylsalicylic acid; $C A B G$, coronary artery bypass grafting; $C I$, confidence interval; euroSCORE, European System for Cardiac Operative Risk Evaluation; $M A C E$, major adverse cardiac events; $P C I$, percutaneous coronary intervention. *Multivessel coronary disease was intended as 3-vessel or 2-vessel plus left main disease.

mortality (odds ratio [OR] $2.1 ; 95 \%$ confidence interval $[\mathrm{CI}], 1.1-3.5 ; P=.001)$ and MACE $(\mathrm{OR}, 1.6 ; 95 \% \mathrm{CI}$, $1.1-2.3 ; \quad P=.006)$. Conditional logistic regression analysis confirmed that continuative statin therapy after previous PCI was significantly associated with reduced risk for hospital mortality (OR, 0.27; 95\% CI, 0.12-0. $57 ; P=.004)$ and $\mathrm{MACE}(\mathrm{OR}, 0.31 ; 95 \% \mathrm{CI}$, $0.18-0.78 ; P=.003)$.

Two-year follow-up was $95 \%$ complete for mortality and $88 \%$ complete for MACE in the propensity score-matched population. Cardiac deaths and MACE at 2 years were significantly lower in the statin group than in the no statin group $(3.4 \%$ vs $5.3 \%, P=.04$ and $5.5 \%$ vs $10.4 \%$, $P=.006$ ). Conditional logistic regression analysis confirmed that continuative statin therapy after previous PCI was significantly associated with reduced risk for 2-year mortality (OR, 0.6; 95\% CI, 0.36-0.96; $P=.04$ ) and MACE (OR, $0.5 ; 95 \%$ CI, 0.34-0.76; $P=.006$ ). Moreover, all demographic and clinical variables listed in Table 1 were analyzed by Cox regression model for their effect on 2-year survival and MACE (Table 5).

\section{DISCUSSION}

Our results demonstrate that patients with a history of PCI with stenting who were treated by continuative statin therapy before elective primary CABG had a significantly lower risk of operative mortality and MACE and better survival and freedom from MACE at 2 years follow-up compared with untreated patients.
After risk adjustment in a multivariate regression model, age 70 years or more, female sex, ejection fraction 0.40 or less, hypertension, diabetes mellitus, previous MI, 3-vessel or 2-vessel plus left main disease, logistic European System for Cardiac Operative Risk Evaluation 5 or greater, multiple previous PCI, and aortic crossclamp time 80 minutes or more in patients undergoing operation with $\mathrm{CPB}$ were independent preoperative markers of deaths and complications. By contrast, the type of stent implanted did not result in significant detrimental effects on hospital mortality and MACE. Of note, perioperative leakage of troponin I was significantly lower in statin-treated patients and was an independent predictor of hospital MACE and 2-year deaths and MACE. This finding is consistent with data from recent literature reporting that an absolute reduction of perioperative troponin I release would translate into a reduction of surgical and overall risk after CABG surgery. ${ }^{22}$

After the statin-treated and untreated patients were balanced by propensity score matching, the benefits conferred by continuative statin therapy on both mortality and MACE were confirmed by an approximately 2 -fold risk reduction.

The exact mechanisms underlying these results are unclear and cannot be elucidated from this study. It is likely that statin pleiotropic properties could attenuate the chronic inflammation and focal trauma secondary to PCI, both of which play an important role in the evolution of coronary artery disease. ${ }^{23-26}$ This hypothesis is supported by Laufs and coworkers, ${ }^{27}$ who demonstrated that statins enhance endothelial nitric oxide (NO) production by directly upregulating endothelial cell NO synthase expression and activity. Thus, by reversing the inhibitory effects of oxidized low-density lipoprotein on endothelial cell NO synthase expression, statins may increase the availability of endothelium-derived NO, which is known to provide the stabilization of the endothelial function and the optimization of local flow. ${ }^{27}$ Finally, it is noteworthy that patients in the statin group displayed a slight, but statistically significant prolonged interval of time from PCI to CABG surgery.

\section{Study Limitations}

The retrospective nature of the analysis represents the main limitation of this study. Nevertheless, despite the use of propensity scoring and multivariate regression models, immeasurable or unknown factors of bias may still exist. The study also could be limited because of the lack of available cardiac catheterization data at the time of PCI, which hampered the evaluation of coronary lesions and the completeness of percutaneous revascularization, both of which are important determinants of outcome after PCI. Moreover, we could not comment on whether the type of stent used had some impact on outcome because different devices with different characteristics 
and indications were implanted in patients over time. Finally, we cannot discuss the specific effectiveness of each statin because different types of statins were used at variable doses and the types of statins were changed in a number of patients between PCI and subsequent CABG.

\section{CONCLUSIONS}

Our results demonstrate a substantial improvement in outcome for statin-treated patients who required subsequent CABG surgery after PCI. This study may provide further evidence in the ongoing discussion of beneficial effects of statin and supports a more extensive use of statins, not only in hyperlipidemic patients, as a long-term treatment after PCI. It is regrettable that although statins are indicated by current guidelines, they are underused in daily practice and long-term adherence to drug regimens is generally poor. Our results suggest careful statin treatment after PCI to fully benefit from the potential of these agents in terms of delayed necessity of repeat surgical revascularization and better outcome when surgical revascularization is required.

\section{References}

1. Barakate MS, Hemli JM, Hughes CF, Bannon PG, Horton MD. Coronary artery bypass grafting $(\mathrm{CABG})$ after initially successful percutaneous transluminal coronary angioplasty (PTCA): a review of 17 years experience. Eur J Cardiothoracic Surg. 2003;23:179-86.

2. Daemen J, Boersma E, Flather M, Booth J, Stables R, Rodriguez A, et al. Long-term safety and efficacy of percutaneous coronary intervention with stenting and coronary artery bypass surgery for multivessel coronary artery disease: a meta-analysis with 5-year patient-level data from the ARTS, ERACI-II, MASS-II, and SoS trials. Circulation. 2008;118:1146-54.

3. Lazar HL. Detrimental effects of coronary stenting on subsequent coronary artery bypass surgery: Is there another flag on the field? J Thorac Cardiovasc Surg. 2009; 138:276-7.

4. Massoudy P, Thielmann M, Lehmann N, Marr A, Kleikamp G, Maleszka A, et al. Impact of prior percutaneous coronary intervention on the outcome of coronary artery bypass surgery: a multicenter analysis. J Thorac Cardiovasc Surg. 2009; 137:840-5.

5. Bonaros N, Hennerbichler D, Friedrich G, Kocher A, Pachinger O, Laufer G, et al. Increased mortality and perioperative complications in patients with previous elective percutaneous coronary interventions undergoing coronary artery bypass surgery. J Thorac Cardiovasc Surg. 2009; 137:846-52.

6. Tran HA, Barnett SD, Hunt SL, Chon A, Ad N. The effect of previous coronary artery stenting on short- and intermediate-term outcome after surgical revascularization in patients with diabetes mellitus. J Thorac Cardiovasc Surg. 2009; 138:316-23

7. Mannacio V, Di Tommaso L, De Amicis V, Lucchetti V, Pepino P, Musumeci F, et al. Previous percutaneous coronary interventions increase mortality and morbidity after coronary surgery. Ann Thorac Surg. 2012;93:1956-63.

8. Smith SC Jr, Allen J, Blair SN, Bonow RO, Brass LM, Fonarow GC, et al, AHA/ACC guidelines for secondary prevention for patients with coronary and other atherosclerotic vascular disease: 2006 update: endorsed by the National Heart, Lung, and Blood Institute. Circulation. 2006;113:2363-72.

9. Mannacio V, Di Tommaso L, Antignano A, De Amicis V, Vosa C. Aspirin plus clopidogrel for optimal platelet inhibition following off-pump coronary artery bypass surgery: results from the CRYSSA (prevention of Coronary arteRY bypaSS occlusion after off-pump procedures) randomised study. Heart. 2012; 98:1710-5.

10. Gurbuz AT, Zia AA, Vuran AC, Cui H, Aytac A. Postoperative clopidogre improves mid-term outcome after off-pump coronary artery bypass graft surgery: a prospective study. Eur J Cardiothorac Surg. 2006;29:190.e5.

11. Gao G, Zheng Z, Pi Y, Lu B, Lu J, Hu S. Aspirin plus clopidogrel therapy increases early venous graft patency after coronary artery bypass surgery a single-center, randomized, controlled trial. J Am Coll Cardiol. 2010;56: 1639.e43.

12. Patti G, Cannon CP, Murphy SA, Mega S, Pasceri V, Briguori C, et al Clinical benefit of statin pretreatment in patients undergoing percutaneous coronary intervention: a collaborative patient-level meta-analysis of 13 randomized studies. Circulation. 2011;123:1622-32.

13. Liakopoulos OJ, Choi YH, Haldenwang PL, Strauch J, Wittwer T, Dörge H, et al Impact of preoperative statin therapy on adverse postoperative outcomes in patients undergoing cardiac surgery: a meta-analysis of over 30,000 patients Eur Heart J. 2008;29:1548-59.

14. Clark LL, Ikonomidis JS, Crawford FA Jr, Crumbley A III, Kratz JM, Stroud MR, et al. Preoperative statin treatment is associated with reduced postoperative mortality and morbidity in patients undergoing cardiac surgery: an 8-year retrospective cohort study. J Thorac Cardiovasc Surg. 2006;131:679-85.

15. Lazar HL, Bao Y, Zhang Y, Bernard SA. Pretreatment with statins enhance myocardial protection during coronary revascularization. J Thorac Cardiovasc Surg. 2003; 125:1037-42.

16. Mannacio VA, Iorio D, De Amicis V, Di Lello F, Musumeci F. Effect of rosuvastatin pretreatment on myocardial damage after coronary surgery: a randomized trial. J Thorac Cardiovasc Surg. 2008;136:1541-8.

17. Chen WT, Krishnan GM, Sood N, Kluger J, Coleman CI. Effect of statins on atrial fibrillation after cardiac surgery: a duration and dose-response meta-analysis. J Thorac Cardiovasc Surg. 2010;140:364-72.

18. Osterberg L, Blaschke T. Adherence to medication. N Engl J Med. 2005;353: 487-97.

19. Hillis LD, Smith PK, Anderson JL, Bittl JA, Bridges CR, Byrne JG, et al. 2011 ACCF/AHA guideline for coronary artery bypass graft surgery: executive summary: a report of the American College of Cardiology Foundation/American Heart Association Task Force on Practice Guidelines. J Thorac Cardiovasc Surg. 2012;143:4-34.

20. Thygesen K, Alpert JS, Jaffe AS, Simoons ML, Chaitman BR, White HD. The Writing Group on behalf of the Joint ESC/ACCF/AHA/WHF Task Force for the Universal Definition of Myocardial Infarction. Third Universal Definition of Myocardial Infarction. Circulation. 2012;126:2020-35.

21. Mannacio V, Di Tommaso L, De Amicis V, Stassano P, Musumeci F, Vosa C Preoperative intraaortic balloon pump for off-pump coronary arterial revascularization. Ann Thorac Surg. 2012;93:804-9.

22. Fellahi JL, Gue X, Richomme X, Monier M, Guillou L, Riou B. Short and long term prognostic value of troponin I concentration in patients undergoing coronary artery bypass grafting. Anesthesiology. 2003;99:270-4.

23. Guven GS, Atalar E, Yavuz B, Beyazit Y, Kekilli M, Kilicarslan A, et al Simvastatin treatment improves endothelial function and increases fibrinolysis in patients with hypercholestrolemia. J Natl Med Assoc. 2006;98:627-30.

24. Tailor A, Lefer DJ, Granger DN. HMG-CoA reductase inhibitor attenuates platelet adhesion in intestinal venules of hypercholesterolemic mice. Am J Physiol Heart Circ Physiol. 2004;286:H1402-7.

25. Sattar N, Murray HM, McConnachie A, Blauw GJ, Bollen EL, Buckley BM et al. C-reactive protein and prediction of coronary heart disease and global vascular events in the Prospective Study of Pravastatin in the Elderly at Risk (PROSPER). Circulation. 2007;115:981-9.

26. Nissen SE, Nicholls SJ, Sipahi I, Libby P, Raichlen JS, Ballantyne CM, et al. Effect of very high intensity statin therapy on regression of coronary atherosclerosis: the ASTEROID trial. JAMA. 2006;295:1556-65.

27. Laufs U, La Fata V, Plutzky J, Liao JK. Upregulation of endothelial nitric oxide synthase by HMG CoA reductase inhibitors. Circulation. 1998;97:1129-35. 